Review

\title{
Effect of resistance training on muscle strength and rate of force development in healthy older adults: A systematic review and meta-analysis
}

\author{
Pedrode Camargo Guizelini ${ }^{\mathrm{a}}$, Rafael Alves de Aguiar $^{\mathrm{b}}$, Benedito Sérgio Denadai ${ }^{\mathrm{a}}$, \\ Fabrizio Caputo $^{\mathrm{b}}$, Camila Coelho Greco ${ }^{\mathrm{a}, *}$ \\ ${ }^{a}$ Human Performance Laboratory, São Paulo State University - UNESP, Avenida 24A, 1515, Bela Vista, Rio Claro, SP CEP 13506-900, Brazil \\ ${ }^{\mathrm{b}}$ Human Performance Research Group, Center for Health and Sport Sciences, Santa Catarina State University - UDESC, Rua Pascoal Simone, 358, Coqueiros, \\ Florianópolis, SC CEP 88080-350, Brazil
}

\section{A R T I C L E I N F O}

\section{Keywords:}

Aging

Elderly

Resistance training

Explosive training

\begin{abstract}
A B S T R A C T
Rapid force capacity, identified by rate of rise in contractile force at the onset of contraction, i.e., the rate of force development (RFD), has been considered an important neuromuscular parameter of physical fitness in elderly individuals. Randomized control studies conducted in adults have found that resistance training may elicit different outcomes in terms of RFD and muscle strength. Thus, the main purpose of this study was to review systematically the literature for studies regarding the influence of resistance training on muscle strength and RFD in elderly persons. A literature search was performed in major electronic databases from inception to March 2017. Studies including health individuals with a mean age $\geq 60$ years, describing the effect of resistance training on RFD and muscle strength were found eligible. The outcomes were calculated as the difference in percentage change between control and experimental groups (\% change) and data were presented as mean $\pm 95 \%$ confidence limits. Meta-analyses were performed using a random-effects model and, in addition, simple and multiple meta-regression analyses were used to identify effects of age, training type, sessions per week and training duration on \% change in RFD and muscle strength. Thirteen training effects were collected from 10 studies included in the meta-analysis. The resistance training program had a moderate beneficial effect on both muscle strength $(\%$ change $=18.40 \%, 95 \%$ CL $13.69-23.30, p<0.001)$ and RFD $(\%$ change $=26.68$, 95\% CL 14.41-35.52, $p<0.001$ ). Results of the meta-regression revealed that the variables age, training type (i.e., strength and explosive), training duration (4-16 weeks) and sessions per week had no significant effects on muscle strength and RFD improvement. Moreover, there was no significant relationship $(p=0.073)$ between the changes in muscle strength and RFD. It can be concluded that explosive training and heavy strength training are effective resistance training methods aiming to improve both muscle strength and RFD after short-to-medium training period. However, muscle strength and RFD seem to adapt differently to resistance training programs, suggesting caution for their interchangeable use in clinical assessments of the elderly.
\end{abstract}

\section{Introduction}

Aging is associated with decrease in muscle strength, which has been explained by factors such as neural changes (i.e., reduced voluntary muscle activation) (Manini and Clark, 2012) and sarcopenia (i.e., losses in muscle size and function) (Häkkinen et al., 1998; Reeves et al., 2004). Thus, resistance training has been frequently included in the exercise programs aiming to improve muscle function and to maintain independence in the later life. Indeed, some systematic reviews and meta-analyses have shown that resistance training can increase muscle strength, even in very old individuals (Silva et al., 2014; Borde et al., 2015; Straight et al., 2016). However, the increased muscle strength in elderly seems to exert only a small to moderate effect on performance during daily activities such as stair climbing, chair rising and walking (Latham et al., 2004; Liu and Latham, 2009). Thus, other neuromuscular parameters related to muscle function must be investigated aiming to elaborate a more efficient training program for this population.

Rapid force capacity, identified by rate of rise in contractile force at the onset of contraction, i.e., the rate of force development (RFD), has been considered an important neuromuscular parameter during "explosive" muscle actions (e.g. jumping and sprint running) (Maffiuletti et al., 2016). Since the time window (50-250 ms) involved in the "explosive" movements are very short, they may not allow maximal muscle

\footnotetext{
* Corresponding author at: Human Performance Laboratory, UNESP, Av. 24 A, 1515, Bela Vista, Rio Claro, SP CEP 13506-900, Brazil.

E-mail address: grecocc@rc.unesp.br (C.C. Greco).
} 


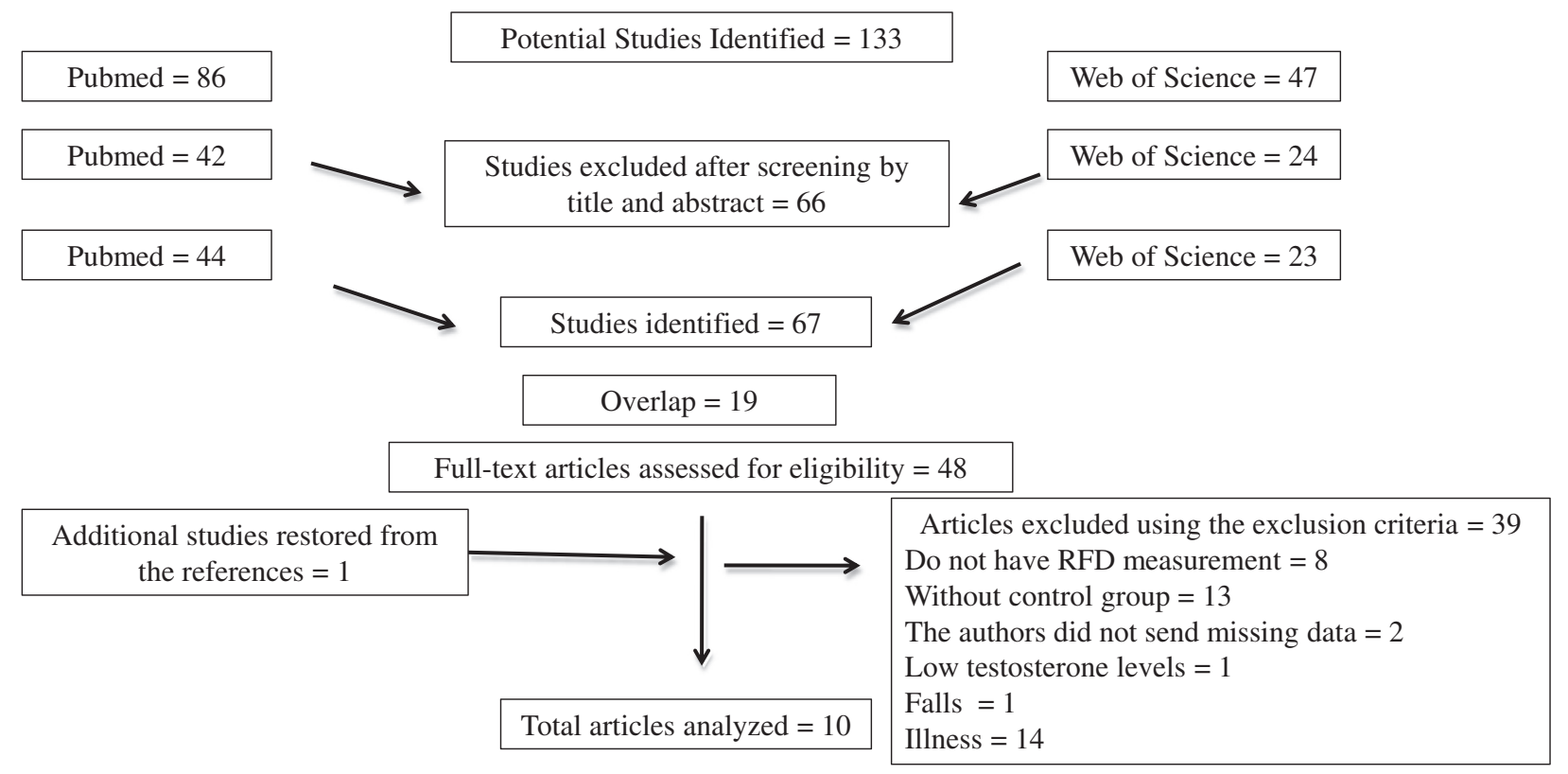

Fig. 1. Flowchart expressing the different phases of the search and selection of the studies included in the meta-analysis.

force to be reached (Aagaard et al., 2010). In addition to the contribution for performance of some sports movements, the RFD has been also considered an important aspect of physical fitness of elderly individuals, since the capacity to rapidly generate force can help during activities of daily living (Aagaard et al., 2010), for balance control (Hess et al., 2006), and to reduce the incidence of falls (Rubenstein, 2006). Although some factors that modulate the strength responses to training are well known in healthy older adults (Silva et al., 2014), much less is known about how specific exercise interventions improve RFD in this population. It is important to note that the resistance training in adults may elicit different outcomes in terms of RFD obtained during the early phase of muscle contraction (i.e., $<100 \mathrm{~ms}$ ) and muscle strength (Oliveira et al., 2013; de Oliveira et al., 2013). Indeed, the mechanisms associated with the RFD early (neural drive, contractile properties and fiber type composition) and RFD late (muscle size, muscle strength, neural drive and the stiffness of the tendonaponeurosis complex) are not the same (Andersen and Aagaard, 2006). Thus, it can be interesting to analyze, in the same healthy old adults, if muscle strength and RFD early respond in a similar manner to the different resistance training programs. In addition to the theoretical implications, this analysis can have important practical application regarding the neuromuscular evaluation and training prescription, since the logistical and resource constraints (i.e., equipment) can limit the widespread determination of RFD as a pragmatic measurement.

Thus, the main purpose of this study was to review systematically the literature for studies regarding the influence of resistance training on muscle strength and RFD in elderly persons. Furthermore, the present meta-analysis, using meta-regression, examines how specific resistance training variables affect muscle strength and RFD. We hypothesized that resistance training can promote different effects on measures of muscle strength and RFD in healthy old adults.

\section{Material and methods}

\subsection{Literature search}

We performed a computerized systematic literature search up to March 2017, for Pubmed and Web of Science databases, including all peer-reviewed longitudinal studies that analyzed the effects of resistance training on the muscle strength and RFD. The following Boolean search strategy was applied using the operators 'and' and 'or': 'elderly', 'aged', 'aging', 'rate of torque development', rate of force development', 'strength development rate', 'weight training', 'resistance training', 'weight bearing exercise program' and 'strength training'. Reference lists of the included articles were checked in an effort to identify suitable studies inclusion in the database. Attempts were also made to contact the authors of the selected articles to request any missing relevant information. This systematic review and meta-analysis is reported in accordance with the preferred reporting items for systematic reviews and meta-analyses (PRISMA) statement (Moher et al., 2009).

\subsection{Inclusion and exclusion criteria}

Resistance training was characterized as exercises where the subject exerted an effort against an external resistance. Studies meeting the following inclusion criteria were considered for review: 1) Available in English; 2) Randomized control studies; 3) Studies that included a test to determine maximal strength and RFD pre- and post-training, and 4) Individuals aged $\geq 60$ years (mean age). The exclusion criteria were used in the selected studies: 1) Review; 2) Illness; 3) Participants with low testosterone levels; 4) Falls; 5) Did not send the data after e-mail contact.

\subsection{Coding of studies}

A search of electronic databases and a scan of article reference list revealed 133 potential studies (Fig. 1). Based on a review of the title or abstract, 66 articles were excluded. Sixty-seven studies were identified. Nineteen studies were excluded by duplicate. Forty-eight studies were assessed for eligibility. One study was restored from the references. Thirty-nine studies were excluded using the exclusion criteria. Ten fulltext articles were included for the meta-analysis.

The outcome measurements were muscle strength and RFD. In all studies, both muscle strength and RFD were measured during isometric contraction, performed in the knee extensors (nine studies) and plantar flexors (one study). If RFD was measured at different times from the onset of contraction, only the highest value was considered for analysis. When a study yielded multiple weight training programs, multiple effects (i.e., heavy strength training and explosive training) were calculated and included separately. The training programs were classified as: 1) Heavy strength training: exercises using $\leq 10$ repetitions and/or 
Table 1

Characteristics of the studies analyzing the effect of training on muscle strength and rate of force development, included in the meta-analysis.

\begin{tabular}{|c|c|c|c|c|c|c|c|c|c|c|}
\hline \multirow[b]{2}{*}{ Study } & \multicolumn{2}{|l|}{ Subjects } & \multicolumn{8}{|c|}{ Training characteristics } \\
\hline & $\mathrm{N}$ & $\begin{array}{l}\text { Age } \\
\text { (yr) }\end{array}$ & Type & Duration (wk) & Sessions per week & $\begin{array}{l}\text { Intensity } \\
\text { (\%1RM) }\end{array}$ & Rep & Sets & $\begin{array}{l}\text { Rep duration } \\
\text { (s) }\end{array}$ & $\begin{array}{l}\text { Rest } \\
\text { (min) }\end{array}$ \\
\hline LaRoche et al. (2008) & $\begin{array}{l}T=12 \\
\mathrm{C}=12\end{array}$ & $\begin{array}{l}71.3 \\
73.7\end{array}$ & Exp & 8 & 3 & NA & 8 & 3 & $0.45-2 \mathrm{CON}$ & 1 \\
\hline Lopes et al. (2014) & $\begin{array}{l}T=13 \\
T=11 \\
\mathrm{C}=11\end{array}$ & $\begin{array}{l}67 \\
63 \\
65\end{array}$ & $\begin{array}{l}\text { HST } \\
\text { Exp }\end{array}$ & $\begin{array}{l}12 \\
12\end{array}$ & $\begin{array}{l}3 \\
3\end{array}$ & $\begin{array}{l}80 \\
40\end{array}$ & $\begin{array}{l}7 \\
5.5\end{array}$ & $\begin{array}{l}2.5 \\
2.5\end{array}$ & $\begin{array}{l}2 \mathrm{CON}, 2 \text { ECC, } \\
\text { CON - H-RFD, } 2 \text { ECC }\end{array}$ & $\begin{array}{l}\text { NA } \\
\text { NA }\end{array}$ \\
\hline Caserotti et al. (2008) & $\begin{array}{l}T=20 \\
\mathrm{C}=20 \\
\mathrm{~T}=12 \\
\mathrm{C}=13\end{array}$ & $\begin{array}{l}62.7 \\
81.8\end{array}$ & $\begin{array}{l}\text { HST } \\
\text { HST }\end{array}$ & $\begin{array}{l}12 \\
12\end{array}$ & 2 & 77.5 & 9 & 4 & $\begin{array}{l}\text { CON - H-RFD } \\
\text { ECC - S-M }\end{array}$ & NA \\
\hline Lovell et al. (2010) & $\begin{array}{l}\mathrm{T}=12 \\
\mathrm{C}=12\end{array}$ & $\begin{array}{l}75 \\
73\end{array}$ & HST & 16 & 3 & 70 & 8 & 3 & NA & 2 \\
\hline Lopes et al. (2016) & $\begin{array}{l}T=14 \\
\mathrm{~T}=12 \\
\mathrm{C}=11\end{array}$ & $\begin{array}{l}69 \\
67 \\
65\end{array}$ & $\begin{array}{l}\text { HST } \\
\text { Exp }\end{array}$ & $\begin{array}{l}12 \\
12\end{array}$ & $\begin{array}{l}3 \\
3\end{array}$ & $\begin{array}{l}60 \\
60\end{array}$ & $\begin{array}{l}8 \\
3.5\end{array}$ & $\begin{array}{l}3 \\
7\end{array}$ & $\begin{array}{l}1 \mathrm{CON}, 1 \mathrm{ECC} \\
\mathrm{CON}-\mathrm{H}-\mathrm{RFD}, 1 \mathrm{ECC}\end{array}$ & $\begin{array}{l}1 \\
3\end{array}$ \\
\hline Bento and Rodacki (2015) & $\begin{array}{l}T=16 \\
\mathrm{C}=16\end{array}$ & $\begin{array}{l}67 \\
66\end{array}$ & HST & 12 & 3 & $\begin{array}{l}\text { NA } \\
10\end{array}$ & 10 & 2.5 & NA & 2 \\
\hline Frank et al. (2016) & $\begin{array}{l}\mathrm{T}=12 \\
\mathrm{C}=10\end{array}$ & $\begin{array}{l}71 \\
72\end{array}$ & HST & 8 & 3 & 77.5 & 12 & 13 & NA & NA \\
\hline Gurjão et al. (2012) & $\begin{array}{l}\mathrm{T}=11 \\
\mathrm{C}=10\end{array}$ & $\begin{array}{l}61 \\
65\end{array}$ & HST & 8 & 3 & $\begin{array}{l}\text { NA } \\
11\end{array}$ & 11 & 3 & $2 \mathrm{CON}, 3 \mathrm{ECC}$ & 1.25 \\
\hline Kobayashi et al. (2016) & $\begin{array}{l}T=17 \\
C=14\end{array}$ & 72 & Exp & 4 & 2 & NA & 10 & 3 & $<1$ ISO & 1 \\
\hline & $\begin{array}{l}T=15 \\
\mathrm{C}=10\end{array}$ & 70 & Exp & 4 & 2 & NA & 10 & 3 & $<1$ ISO & 1 \\
\hline Sundstrup et al. (2016) & $\begin{array}{l}T=9 \\
C=8\end{array}$ & $\begin{array}{l}69 \\
67\end{array}$ & HST & 16 & 2 & $75 \%$ & 10 & 3.5 & NA & 1.5 \\
\hline
\end{tabular}

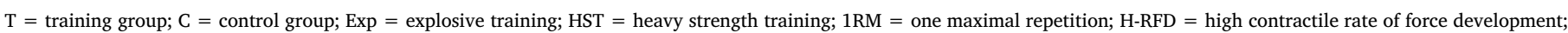
$\mathrm{S}-\mathrm{M}$ = slow-to-moderate velocity; Rep = repetitions; CON = concentric; ECC = eccentric; ISO = isometric. NA - not assigned.

intensity $\geq 70 \% 1 \mathrm{RM}$, without the intention to perform high contractile RFD, and; 2) Explosive training: the subjects performed the exercises with high contractile RFD, irrespectively of the exercise intensity, and/ or number of repetitions. It is important to note that Sundstrup et al. (2016) measured RFD and maximal strength at baseline and after 4 and 12 months post training. However, since every other studies underwent training of up to 16 weeks, the present meta-analysis only used the measurements of 4 months post training. The variables analyzed were age (mean value), training type (heavy strength training vs. explosive training), sessions per week (mean value) and training duration.

\subsection{Study characteristics}

Thirteen training effects were collected from 10 studies included in meta-analysis and their characteristics are summarized in Table 1. A total of 333 subjects were included in the 10 studies: 186 and 147 subjects participated of the control and intervention groups, respectively. The training effects for strength and explosive training were nine and four, respectively. The values expressing muscle strength were determined as $\mathrm{N}, \mathrm{N} \cdot \mathrm{m}, \mathrm{N} \cdot \mathrm{m} \cdot \mathrm{kg}^{-1}$ and $\mathrm{kg}$, and RFD was determined as and $\mathrm{N} \cdot \mathrm{m} \cdot \mathrm{s}^{-1}, \mathrm{~N} \cdot \mathrm{m} \cdot \mathrm{s}^{-1} \cdot \mathrm{kg}^{-1}$ or $\mathrm{N} \cdot \mathrm{s}^{-1}$.

\subsection{Study quality}

The methodological quality of the selected articles was assessed by two authors, using the Physiotherapy Evidence-Based Database Scale (PEDro). The scale yields a total possible score of 11 points, with more points corresponding to higher quality (Maher et al., 2003). A consensus between researchers was reached regarding the final scores of all selected articles.

\subsection{Data extraction}

The numbers of participants, means and standard deviations of the outcome measurements (i.e. muscle strength and RFD) were extracted for both experimental and control conditions. In addition, the $p$ value of the interaction between the control and intervention groups was extracted when presented. Graph digitizer software (Digitizelt, Germany) was used to obtain data values in studies where only plots were published. Accuracy was confirmed via intra- and inter-individual reassessments of data extraction.

To estimate the magnitude of impact of resistance training on outcome measures (i.e. muscle strength and RFD), the difference in changes from baseline between control and experimental groups (i.e., $\%$ change) was calculated. However, while most studies had large percent effects ( $>10 \%$ ), the $\%$ change was expressed as factors and log-transformed before meta-analysis (Hopkins, 2004). For each \% change, standard errors were calculated to indicate the level of imprecision. In studies where exact $p$ values were given $(n=5$ for RFD and muscle strength), standard errors were calculated directly via the corresponding $\mathrm{F}$ value and its degrees of freedom. Based on the assumption that studies with similar test protocols and subject characteristics would have similar typical errors of measurement, the typical errors from these studies were then averaged (via the weighted mean variance) and assigned to the studies that did not report an exact $p$ value. The standard error was then calculated via the relationship between typical error and standard error (Vandenbogaerde and Hopkins, 2011; Carr et al., 2011).

\subsection{Data analysis}

Statistical analyses were performed with Comprehensive MetaAnalysis (CMA) version 3.0 (Englewood, New Jersey, USA) and using a statistical significance set at $p<0.05$. Data were reported as mean $\pm 95 \%$ confidence limits (CL). The overall effect was calculated with a random effects model that accounted for true inter-study variations in effects as well as for random errors within each study (Borenstein et al., 2009). A random-effects model was chosen over a 
fixed-effect model because of the wide variation in experimental factor levels used in the reviewed studies. After analysis, the mean effect estimates and its corresponding 95\% confidence limits were all converted into exact percentages: $100[\exp (\mathrm{x} / 100)]-100$, where $\mathrm{x}$ is the change in the mean, or the lower/upper confidence bound (Hopkins et al., 2009).

To determine the influence of moderating variables on the $\%$ change in muscle strength and RFD, random-effects meta-regressions were performed using one or more than one priori identified variable. While the simple meta-regression (i.e. only one covariate) determine the independent effects of moderator variables on variation in $\%$ change, the meta-regressions using more than one covariate yield a set of statistics for each covariate, as well as set of statistics for the model. The statistics for each covariate reflect the impact of that covariate partialling out the effects of all other covariates in the model. The statistics for the full model reflect the combined impact of all covariates.

We used a scale based upon the one proposed by Hopkins et al. (2009) to evaluate the relative magnitude of the \% change of training research. Accordingly, our inferences were based on standardized thresholds for small, moderate and large changes of $0.2,0.6$ and 1.2 SDs, respectively, and derived by averaging appropriate between-subject variances for baseline RFD and muscle strength. Therefore, for RFD and muscle strength, magnitude thresholds were 7.758, 23.25 and 46.49 and 5.13, 15.40 and $30.80 \%$, respectively.

\subsection{Heterogeneity and publication bias}

The effect of publication bias on the primary meta-analyses was addressed by combining a funnel plot assessment using Duval and Tweedie's trim and fill correction (Rothstein et al., 2005). Statistical heterogeneity, which refers to the percentage of the variability between studies that is due to clinical and methodological heterogeneity rather than the sampling error, was assessed by the $\mathrm{I}^{2}$ statistic (Borenstein et al., 2009). According to Higgins et al. (2003), $\mathrm{I}^{2}$ values of $25 \%, 50 \%$, and $75 \%$ represent low, medium, and high heterogeneity, respectively. In addition, we calculated the standard deviation of true value of the $\%$ change between studies $(T)$.

\section{Results}

The studies included in this meta-analysis analyzed the effect of heavy strength training and explosive training on muscle strength and RFD. The PEDro quality scores for the 10 studies were good and very similar, ranging from 6 to 7 points. Table 1 summarizes the characteristics of the studies analyzing the effect of training on muscle strength and RFD, included in the meta-analysis.

\subsection{Rate of force development}

The effects of training after back transformation varied from 0.27 to $58.11 \%$ for RFD, and the thirteen training effects showed an increase of 26.68 (95\% CL 14.41-35.52, $p<0.001$ ) in RFD after back transformation. The funnel plot placed a disproportionate number of studies to the right of the overall \% change. Using Duval and Tweedie's trim and fill correction, three studies required input into the analysis to produce symmetry around the mean \% change. The results of this correction yielded an overall \% change of $19.91 \%$ (95\% CL 12.36-27.46, $p<0.001$ ) after back transformation, which now represents a significant small beneficial effect. The significant improvement in RFD after the training period was homogenous $(\mathrm{Q}=14.09 ; p=0.29)$ with low inconsistency of effects ( $\mathrm{I}^{2}=14.85 \% ; T=4.73 \%$ ) (Fig. 2).

The simple meta-regression was not significant when moderating by age, training type, sessions per week and training duration (Table 2). However, when moderated by training types, both explosive $(26.89 \%$, 95\% CL 11.29 to $44.67, p<0.001)$ and heavy strength $(27.09 \%, 95 \%$ CL 16.71 to $38.40, p<0.001$ ) training presented a \% change significantly higher than zero.

The results of the meta-regression using multiple predictors, i.e., age, training type, sessions per week and training duration, did not reduce the inconsistency of $\%$ change. In addition, the impact of each covariate partialing out the effects of the other covariates was not significant for any predictors (Table 3 ).

Finally, although the \% change in muscle strength reduced the inconsistency of the \% change in RFD for $0 \%$, there was no significant relationship between the increase in muscle strength and RFD ( $\beta=0.85 \%, 95 \%$ CL -0.08 to $1.79, p=0.073$ ) (Fig. 4 ).

\subsection{Muscle strength}

The effects of training after back transformation varied from 0.60 to $26.87 \%$ on muscle strength, and the thirteen training effects showed an improvement of $18.40 \%$ (95\% CL 13.69 to $23.30, p<0.001$ ) after back transformation, which represent a significant moderate beneficial effect. The funnel plot did not show a disproportionate number of studies for either side of the mean of the overall \% change. The significant improvement in muscle strength after the training period was homogenous $(\mathrm{Q}=10.03 ; p=0.613$ ) with no inconsistency of effects $\left(\mathrm{I}^{2}=0 \% ; T=0 \%\right.$ ) (Fig. 3). In this case, the variability in \% change estimates is due to sampling error within studies.

The simple meta-regression was not significant when moderating by the age, training type, sessions per week and training duration (Table 2). However, when moderated by training types, both explosive (14.53\%, 95\% CL 5.41 to 24.44, $p<0.001$ ) and heavy strength (19.64\%, 95\% CL 14.20 to $25.33, p<0.001$ ) training presented a \% change significantly higher than zero.

The results of the meta-regression using multiple predictors, i.e., age, training type, sessions per week and training duration, was not significant for any predictors (Table 3 ).

\section{Discussion}

The main objective of this study was to review systematically the literature for studies regarding the influence of resistance training on maximal muscle strength and RFD in elderly persons. We found evidence that the resistance training promotes a significant moderate beneficial effect on RFD and muscle strength in this population. However, no significant relationship between the changes in muscle strength and RFD was found, which suggest that these variables could respond in different magnitudes to resistance training. Therefore, muscle strength and RFD should be tested and trained complementarily in elderly persons.

With basis on the studies included in this meta-analysis, our results demonstrate a significant increase of $18.4 \%$ on muscle strength, with all studies indicating a positive effect of training. This result is slightly smaller when compared with other systematic reviews, which demonstrated increase of $24-33 \%$ on muscle strength in elderly persons (Borde et al., 2015; Peterson et al., 2010; Stewart et al., 2014).

Using a meta-regression, it was verified that training duration has no influence on the strength gains after short-to-medium periods (i.e., 4-16 weeks) of resistance training. Different results have been found in previous systematic reviews analyzing studies with longer training periods (52-53 weeks) (Silva et al., 2014; Borde et al., 2015). These studies verified that longer training durations had a greater effect on strength gains compared to shorter duration protocols. Classically, the strength gains have been explained by neural and morphological adaptations (Folland and Williams, 2007), with the former occurring mainly during the early phase of the training period. In elderly persons, Arnold and Bautmans (2014) demonstrated an overall improvement of $8.8 \%$ on voluntary activation, after $6-12$ weeks of resistance training. Moreover, Simoneau et al. (2006) and Scaglioni et al. (2002) found significant correlation between the changes in voluntary activation and muscle strength in elderly persons. These studies have evidenced that 
Reference

Laroche et al., 2008
Lopes et al., 2014
Caserotti et al., 2008
Lovell et al., 2010
Lopes et al., 2016
Kobayashi et al., 2016
Bento et al., 2015
Lopes et al., 2014
Frank et al., 2015
Sundstroup et al., 2016
Gurjao et al., 2012
Caserotti et al., 2008
Lopes et al., 2016

Training type $\%$ Change $\quad$-Value

$\begin{array}{lr}\text { Exp } & 0.270 \\ \text { HST } & 6.430 \\ \text { HST } & 17.370 \\ \text { HST } & 19.240 \\ \text { HST } & 20.050 \\ \text { Exp } & 21.010 \\ \text { HST } & 28.210 \\ \text { Exp } & 30.730 \\ \text { HST } & 31.310 \\ \text { HST } & 31.490 \\ \text { HST } & 41.900 \\ \text { HST } & 43.120 \\ \text { Exp } & 45.810\end{array}$

Overall effect
Exp \begin{tabular}{l|}
0.983 \\
0.610 \\
0.002 \\
0.127 \\
0.105 \\
0.032 \\
0.010 \\
0.020 \\
0.017 \\
0.041 \\
0.009 \\
0.002 \\
0.000 \\
0.000
\end{tabular}

$-70.00$

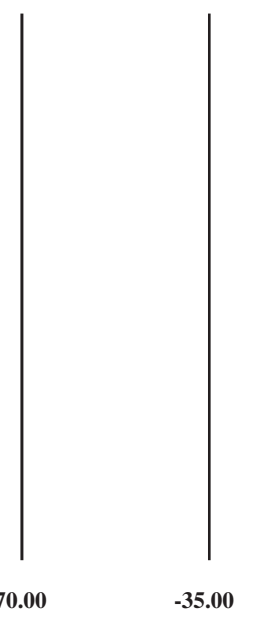

Harmful effects following training
$\%$ Change and $95 \% \mathrm{CI}$

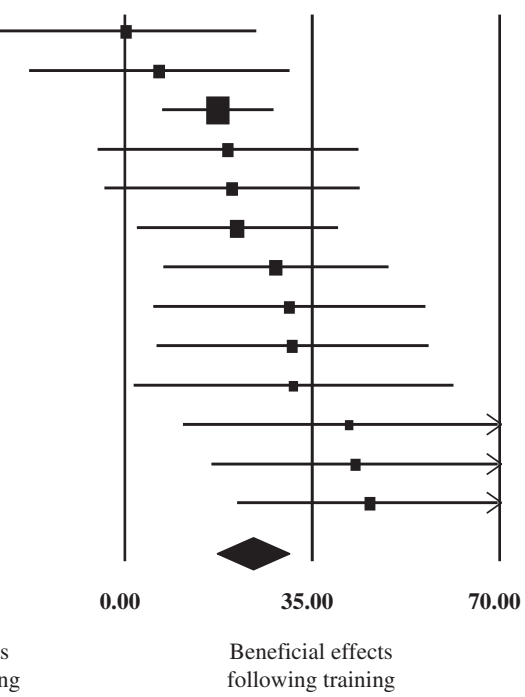

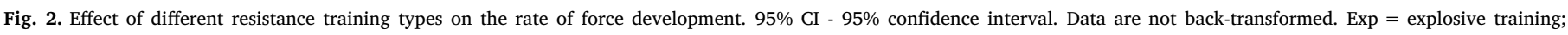
HST $=$ heavy strength training.

Table 2

Effect of training on rate of force development and muscle strength according to age, training type, sessions per week and training duration.

\begin{tabular}{|c|c|c|c|c|c|c|}
\hline & \multicolumn{3}{|c|}{ Rate of force development } & \multicolumn{3}{|c|}{ Muscle strength } \\
\hline & Coefficient & $95 \% \mathrm{CI}$ & $p$ value & Coefficient & $95 \% \mathrm{CI}$ & $p$ value \\
\hline Age & 0.01 & $\begin{array}{l}-1.37 \text { to } \\
1.41\end{array}$ & 0.984 & 0.13 & $\begin{array}{l}-0.55 \\
\text { to } 0.81\end{array}$ & 0.716 \\
\hline $\begin{array}{r}\text { Training } \\
\text { type }\end{array}$ & 0.16 & $\begin{array}{l}-14.34 \\
\text { to } 17.12\end{array}$ & 0.984 & 4.46 & $\begin{array}{l}-5.02 \\
\text { to } 14.89\end{array}$ & 0.3688 \\
\hline $\begin{array}{l}\text { Sessions per } \\
\text { week }\end{array}$ & 1.39 & $\begin{array}{l}-12.83 \\
\text { to } 17.93\end{array}$ & 0.858 & -0.24 & $\begin{array}{l}-8.46 \\
\text { to } 8.73\end{array}$ & 0.957 \\
\hline $\begin{array}{c}\text { Training } \\
\text { dura- } \\
\text { tion }\end{array}$ & 0.38 & $\begin{array}{l}-1.85 \text { to } \\
2.67\end{array}$ & 0.740 & 1.01 & $\begin{array}{l}-0.38 \\
\text { to } 2.42\end{array}$ & 0.155 \\
\hline
\end{tabular}

Back transformed data are shown. CI - confidence interval.

Table 3

Multiple meta-regression analysis including age, training type, sessions per week and training duration.

\begin{tabular}{|c|c|c|c|c|c|c|}
\hline & \multicolumn{3}{|c|}{ Rate of force development } & \multicolumn{3}{|c|}{ Muscle strength } \\
\hline & Coefficient & $95 \% \mathrm{CI}$ & $p$ value & Coefficient & $95 \% \mathrm{CI}$ & $p$ value \\
\hline Intercept & 33.24 & $\begin{array}{l}-65.45 \\
\text { to } 413.87\end{array}$ & 0.677 & 0.37 & $\begin{array}{l}-43.72 \\
\text { to } 78.99\end{array}$ & 0.990 \\
\hline Age & -0.09 & $\begin{array}{l}-1.77 \text { to } \\
1.62\end{array}$ & 0.917 & 0.09 & $\begin{array}{l}-0.62 \text { to } \\
0.79\end{array}$ & 0.811 \\
\hline $\begin{array}{l}\text { Training } \\
\text { type }^{\mathrm{a}}\end{array}$ & -0.88 & $\begin{array}{c}-20.47 \\
\text { to } 23.54\end{array}$ & 0.938 & 1.96 & $\begin{array}{l}-8.30 \text { to } \\
13.37\end{array}$ & 0.720 \\
\hline $\begin{array}{l}\text { Sessions } \\
\text { per } \\
\text { week }\end{array}$ & -1.06 & $\begin{array}{c}-19.04 \\
\text { to } 20.91\end{array}$ & 0.817 & -0.1 & $\begin{array}{l}-8.57 \text { to } \\
9.16\end{array}$ & 0.983 \\
\hline $\begin{array}{c}\text { Training } \\
\text { dura- } \\
\text { tion }\end{array}$ & 0.51 & $\begin{array}{l}-2.70 \text { to } \\
3.84\end{array}$ & 0.758 & 0.87 & $\begin{array}{l}-0.67 \text { to } \\
2.44\end{array}$ & 0.269 \\
\hline
\end{tabular}

Back transformed data are shown.

${ }^{a}$ Heavy strength training was chosen as the reference group.

neural changes contribute to the increase of muscle strength after resistance training performed by elderly. During shorter periods of training (i.e., up to 6 weeks), enhanced voluntary activation has been associated with the increased firing rate of motor units (Gabriel et al.,

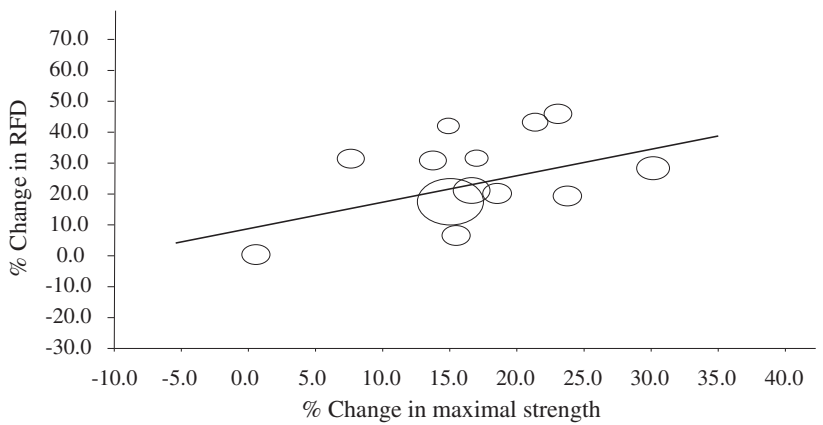

Fig. 4. Correlation between the changes in rate of force development and muscle strength after the training period.

2006), especially during the early stage of the muscle contraction (Van Cutsem et al., 1998). Traditionally, muscle growth and increased muscle-tendon unit stiffness are expected in long-term resistance training programs (Moritani and DeVries, 1979). However, some recent studies conducted in elderly persons showed that resistance training enhances indices associated with muscle hypertrophy (i.e., cross-sectional area and muscle thickness) after 3-9 weeks (Lixandrão et al., 2016; Loenneke et al., 2017). Moreover, Borde et al. (2015) reported that the training period (range 6-52 weeks) has no influence on measures of muscle morphology. Thus, the influence of muscle hypertrophy on strength improvement after long-term training programs performed by elderly people remains to be clearly established.

Previous meta-analyses have produced mixed data in relation to the effect of the resistance training intensity on muscle strength improvement. Peterson et al. (2010) and Silva et al. (2014) compared studies with intensities between 40 and 85\%1RM and verified that higher intensity training was associated with greater improvement on muscle strength. However, Borde et al. (2015), analyzing a broader intensity range (from $50 \% 1 \mathrm{RM}$ to $\geq 90 \% 1 \mathrm{RM}$ ), demonstrated that the effect of intensity is characterized by an inversed U-shape profile. In this study, the intensities between 70 and $79 \% 1 \mathrm{RM}$ generated greater strength gains than higher and lower intensities. It is important to recognize that other training variables (number of sets, rest periods), besides the training intensity, can be important for the muscle strength improvement. Indeed, Csapo and Alegre (2016) found a smaller difference of the muscle strength gain between high- and low-intensity training 


\begin{tabular}{llrl} 
Reference & Training type & \% Change & p-Value \\
Laroche et al., 2008 & Exp & 0.600 & 0.944 \\
Frank et al., 2015 & HST & 7.670 & 0.388 \\
Lopes et al., 2014 & Exp & 13.780 & 0.121 \\
Gurjao et al., 2012 & HST & 14.930 & 0.009 \\
Caserotti et al., 2008 & HST & 15.090 & 0.002 \\
Lopes et al., 2014 & HST & 15.510 & 0.069 \\
Kobayashi et al., 2016 & Exp & 16.670 & 0.034 \\
Sundstroup et al., 2016 & HST & 17.030 & 0.103 \\
Lopes et al., 2016 & HST & 18.570 & 0.026 \\
Caserotti et al., 2008 & HST & 21.400 & 0.002 \\
Lopes et al., 2016 & Exp & 23.100 & 0.008 \\
Lovell et al., 2010 & HST & 23.800 & 0.005 \\
Bento et al., 2015 & HST & 30.190 & 0.000 \\
\multicolumn{1}{c}{ Overall effect } & & 16.888 & 0.000
\end{tabular}

$\%$ Change and $95 \%$ CI

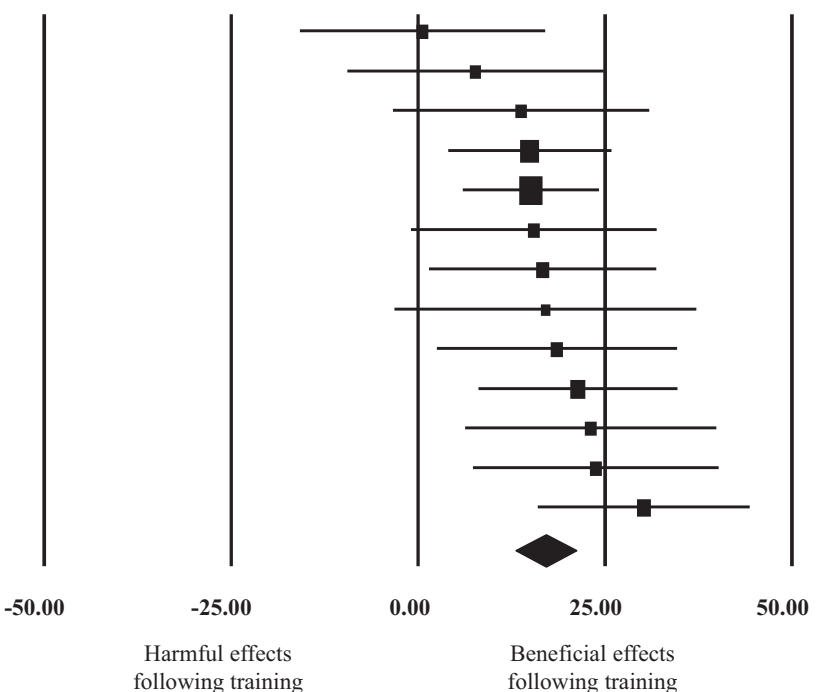

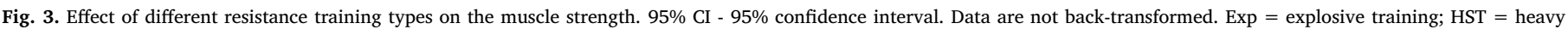
strength training.

programs, when they were matched for mechanical work. In the present study, the training type did not have effect on muscle strength gains, when the training was performed using high-intensity and low velocity of contraction or low-intensity and high velocity of contraction. Our systematic search identified two studies that directly compared resistance training protocols of different intensities (Lopes et al., 2014, 2016). In both studies, the different training types (i.e., heavy strength training and power training) induced to similar increase in muscle strength. These data suggest that, in elderly persons, high loads are not necessary to induce the greater muscle strength gains. On the other hand, the velocity of contraction could be an important factor involved in strength gains. Indeed, the velocity of contraction is determinant to induce some neural adaptations (Behm and Sale, 1993) that are essential for the strength gains, especially during the first weeks of training for elderly persons. However, we recognize that the inclusion criteria utilized in the present meta-analysis, determined a relative low number of randomized-controlled trials, which did not permit a more detailed analysis about the dose-response relationship for the development of the muscle strength. Finally, although there is an important age-related loss of muscle strength, the strength gain was similar among subjects in the age range analyzed. Indeed, Cadore et al. (2014) have demonstrated that nonagenarians can improve muscle strength with training (20\%).

To our knowledge, this is the first study that analyzed the effect of different types of resistance training on RFD in elderly persons. With basis on the studies included in this meta-analysis, the resistance training promoted a significant increase of $26.68 \%$ on RFD, with the majority of studies indicating a positive effect of training. In addition, together with no significant heterogeneity, there was only low inconsistency of the effects among studies, suggesting little or no influence of clinical and methodological variability on \% change in RFD in healthy old adults.

The training type and age did not have influence on the changes in RFD, as was for muscle strength. In adults, different resistance training types (i.e., explosive, heavy strength training), have promoted significant gains in contractile RFD (Oliveira et al., 2013; de Oliveira et al., 2013). However, Young and Bilby (1993) have demonstrated that resistance training performed with high velocity of contraction induced to a greater increase on RFD, than that conducted using low velocity of contraction. Interestingly, the magnitude of the RFD enhancement seems to be independent of the ballistic-intended movements in elderly persons. It is important to emphasize that the maximal limb velocity during the explosive training can be highly variable in elderly persons (Sayers et al., 2016), which could theoretically attenuate the traininginduced changes in RFD.

Similar to that observed for muscle strength, contractile RFD was not moderated by training duration. Additionally, the training-induced changes in muscle strength and RFD were not significantly correlated. Thus, muscle strength and early RFD seem not fully share the same mechanisms in elderly persons, as previously demonstrated by interventional (e.g., resistance training and muscle damage) (Oliveira et al., 2013; de Oliveira et al., 2013; Molina and Denadai, 2012) and crosssectional studies performed in adults (Andersen and Aagaard, 2006). Andersen and Aagaard (2006) found that the correlations between muscle strength (maximal voluntary isometric contraction) and RFD early ( $<100 \mathrm{~ms}$ ) become smaller $(r=0.75$ to 0.42 ) with decreasing time windows for RFD analyses, suggesting that the longer is the RFD time from the peak strength, the lower is the relationship between the strength and RFD early. Although highly influenced by neural drive (Gruber and Gollhofer, 2004), the RFD early seems to be also dependent of intrinsic muscle contractile properties (i.e., fiber type and myosin heavy chain composition - MHC) (Andersen and Aagaard, 2006). Classically, resistance training-induced changes in the neural drive are observed mainly during the first weeks of training (e.g., 2-8 weeks) (Maffiuletti et al., 2016). However, myosin heavy chain IIX isoforms (an important determinant of muscle contractile properties) are likely to be reduced (50-90\%) during prolonged resistance training protocols (Andersen et al., 2010). Thus, interactions between neural and muscular mechanisms can help to explain the absence of significant effect of training duration on the RFD changes.

Although the present analysis provides important and novel data, the findings of the review must be considered in the context of a number of limitations. First of all, the poor methodological quality of many trials should be acknowledged. There is evidence that lack of blinding of the outcome assessor, intention to treat-analysis or allocation concealment may exaggerate the effect sizes. Second, the analysis is based on a relatively low number of studies and subjects, which may have contributed to the observed effect sizes in the sub-groups analyzed, as well as the precision of the effects. Finally, most participants involved in these randomized-controlled trials were healthy and untrained, and extrapolating the effect of resistance training on strength and RFD to other populations with pathological conditions and superior 
training status (i.e., physically active or trained) should be done carefully.

\section{Conclusions}

The present meta-analysis shows that resistance training periods lasting 4-16 weeks are effective to promote significant muscle strength and RFD gains in elderly persons, irrespectively of training type (i.e., heavy strength and explosive), age and training duration. However, a non-significant relationship was found between the training-induced gains in muscle strength and RFD, suggesting that these neuromuscular parameters should not be used interchangeably for clinical assessments (e.g., risk of falls and balance control) of the elderly. Moreover, in a clinical practice and research muscle strength and RFD should be tested and trained complementarily in this population. Future prospective studies should evaluate a possible dose-response relationship between training-induced changes in RFD and performance during daily activities. In particular, we strongly suggest that maximal limb velocity be carefully controlled during the explosive training, aiming to improve our understanding about the training-induced changes in RFD after this resistance training type.

\section{References}

Aagaard, P., Suetta, C., Caserotti, P., Magnusson, S.P., Kjaer, M., 2010. Role of the nervous system in sarcopenia and muscle atrophy with aging: strength training as a countermeasure. Scand. J. Med. Sci. Sports 20 (1), 49-64. http://dx.doi.org/10. 1111/j.1600-0838.2009.01084.x.

Andersen, L.L., Aagaard, P., 2006. Influence of maximal muscle strength and intrinsic muscle contractile properties on contractile rate of force development. Eur. J. Appl. Physiol. 96 (1), 46-52.

Andersen, L.L., Andersen, J.L., Zebis, M.K., Aagaard, P., 2010. Early and late rate of force development: differential adaptive responses to resistance training? Scand. J. Med. Sci. Sports 20, e162-e169.

Arnold, P., Bautmans, I., 2014. The influence of strength training on muscle activation in elderly persons: a systematic review and meta-analysis. Exp. Gerontol. 58, 58-68. http://dx.doi.org/10.1016/j.exger.2014.07.012.

Behm, D.G., Sale, D.G., 1993. Velocity specificity of resistance training. Sports Med. 15 (6), 374-388.

Bento, P.C., Rodacki, A.L., 2015. Muscle function in aged women in response to a waterbased exercises program and progressive resistance training. Geriatr Gerontol Int 15 (11), 1193-1200. http://dx.doi.org/10.1111/ggi.12418.

Borde, R., Hortobágyi, T., Granacher, U., 2015. Dose-response relationships of resistance training in healthy old adults: a systematic review and meta-analysis. Sports Med. 45 (12), 1693-1720. http://dx.doi.org/10.1007/s40279-015-0385-9.

Borenstein, M., Hedges, L., Higgins, J., Rothstein, H.R., 2009. Introduction to MetaAnalysis, first edition. Wiley, West Sussex.

Cadore, E.L., Casas-Herrero, A., Zambom-Ferraresi, F., Idoate, F., Millor, N., Gómez, M., Rodriguez-Mañas, L., Izquierdo, M., 2014. Multicomponent exercises including muscle power training enhance muscle mass, power output, and functional outcomes in institutionalized frail nonagenarians. Age (Dordr.) 36 (2), 773-785. http://dx.doi. org/10.1007/s11357-013-9586-z.

Carr, A.J., Hopkins, W.G., Gore, C.J., 2011. Effects of acute alkalosis and acidosis on performance: a meta-analysis. Sports Med. 41 (10), 801-814. http://dx.doi.org/10. 2165/11591440-000000000-00000.

Caserotti, P., Aagaard, P., Larsen, J.B., Puggaard, L., 2008. Explosive heavy-resistance training in old and very old adults: changes in rapid muscle force, strength and power. Scand. J. Med. Sci. Sports 18 (6), 773-782. http://dx.doi.org/10.1111/j. 1600-0838.2007.00732.x.

Csapo, R., Alegre, L.M., 2016. Effects of resistance training with moderate vs heavy loads on muscle mass and strength in the elderly: a meta-analysis. Scand. J. Med. Sci. Sports 26 (9), 995-1006. http://dx.doi.org/10.1111/sms.12536.

Folland, J.P., Williams, A.G., 2007. The adaptations to strength training: morphological and neurological contributions to increased strength. Sports Med. 37 (2), 145-168.

Frank, P., Andersson, E., Pontén, M., Ekblom, B., Ekblom, M., Sahlin, K., 2016. Strength training improves muscle aerobic capacity and glucose tolerance in elderly. Scand. J. Med. Sci. Sports 26 (7), 764-773. http://dx.doi.org/10.1111/sms.12537.

Gabriel, D.A., Kamen, G., Frost, G., 2006. Neural adaptations to resistive exercise: mechanisms and recommendations for training practices. Sports Med. 36 (2), 133-149.

Gruber, M., Gollhofer, A., 2004. Impact of sensorimotor training on the rate of force development and neural activation (Eur. J. Appl. Physiol.). 92 (1-2), 98-105. http:// dx.doi.org/10.1007/s00421-004-1080-y.

Gurjão, A.L., Gobbi, L.T., Carneiro, N.H., Gonçalves, R., Ferreira de Moura, R., Cyrino, E.S., Altimari, L.R., Gobbi, S., 2012. Effect of strength training on rate of force development in older women. Res. Q. Exerc. Sport 83 (2), 268-275.

Häkkinen, K., Kallinen, M., Izquierdo, M., Jokelainen, K., Lassila, H., Mälkiä, E., Kraemer, W.J., Newton, R.U., Alen, M., 1998. Changes in agonist-antagonist EMG, muscle CSA, and force during strength training in middle-aged and older people. J. Appl. Physiol.
84 (4), 1341-1349.

Hess, J.A., Woollacott, M., Shivitz, N., 2006. Ankle force and rate of force production increase following high intensity strength training in frail older adults. Aging Clin. Exp. Res. 18 (2), 107-115.

Higgins, J.P., Thompson, S.G., Deeks, J.J., Altman, D.G., 2003. Measuring inconsistency in meta-analyses. BMJ 327 (7414), 557-560.

Hopkins, W.G., 2004. An introduction to meta-analysis. Sportscience 8.

Hopkins, W.G., Marshall, S.W., Batterham, A.M., Hanin, J., 2009. Progressive statistics for studies in sports medicine and exercise science. Med. Sci. Sports Exerc. 41 (1), 3-13.

Kobayashi, Y., Ueyasu, Y., Yamashita, Y., Akagi, R., 2016. Effects of 4 weeks of explosivetype strength training for the plantar flexors on the rate of torque development and postural stability in elderly individuals. Int. J. Sports Med. 37 (6), 470-475. http:// dx.doi.org/10.1055/s-0035-1569367.

LaRoche, D.P., Roy, S.J., Knight, C.A., Dickie, J.L., 2008. Elderly women have blunted response to resistance training despite reduced antagonist coactivation. Med. Sci. Sports Exerc. 40 (9), 1660-1668. http://dx.doi.org/10.1249/MSS. 0b013e3181761561.3.

Latham, N.K., Bennett, D.A., Stretton, C.M., Anderson, C.S., 2004. Systematic review of progressive resistance strength training in older adults. J. Gerontol. A. Biol. Sci. Med. Sci. 59 (1), 48-61.

Liu, C.J., Latham, N.K., 2009. Progressive resistance strength training for improving physical function in older adults. Cochrane Database Syst. Rev. 8 (3), CD002759. http://dx.doi.org/10.1002/14651858.CD002759.pub2.

Lixandrão, M.E., Damas, F., Chacon-Mikahil, M.P., Cavaglieri, C.R., Ugrinowitsch, C., Bottaro, M., Vechin, F.C., Conceição, M.S., Berton, R., Libardi, C.A., 2016. Time course of resistance training-induced muscle hypertrophy in the elderly. J. Strength Cond. Res. 30 (1), 159-163. http://dx.doi.org/10.1519/JSC.0000000000001019.

Loenneke, J.P., Rossow, L.M., Fahs, C.A., Thiebaud, R.S., Grant Mouser, J., Bemben, M.G., 2017. Time-course of muscle growth, and its relationship with muscle strength in both young and older women. Geriatr. Gerontol. Int. http://dx.doi.org/10.1111/ggi. 13010.

Lopes, P.B., Pereira, G., de Souza, D.M., Rodacki, A.L., 2014. Comparison between strength and power training on elderly force-generating ability. Top. Geriatr. Rehabil. 30 (4), 264-269. http://dx.doi.org/10.1097/TGR.0000000000000033.

Lopes, P.B., Pereira, G., Lodovico, A., Bento, P.C., Rodacki, A.L., 2016. Strength and power training effects on lower limb force, functional capacity and static and dynamic balance in older female adults. Rejuvenation Res. 19 (5), 385-393. http://dx. doi.org/10.1089/rej.2015.1764.

Lovell, D.I., Cuneo, R., Gass, G.C., 2010. The effect of strength training and short-term detraining on maximum force and the rate of force development of older men. Eur. J. Appl. Physiol. 109 (3), 429-435. http://dx.doi.org/10.1007/s00421-010-1375-0.

Maffiuletti, N.A., Aagaard, P., Blazevich, A.J., Folland, J., Tillin, N., Duchateau, J., 2016. Rate of force development: physiological and methodological considerations. Eur. J. Appl. Physiol. 116 (6), 1091-1116. http://dx.doi.org/10.1007/s00421-016-3346-6.

Maher, C.G., Sherrington, C., Herbert, R.D., Moseley, A.M., Elkins, M., 2003. Reliability of the PEDro scale for rating quality of randomized controlled trials. Phys. Ther. 83 (8), 713-721.

Manini, T.M., Clark, B.C., 2012. Dynapenia and aging: an update. J. Gerontol. A Biol. Sci. Med. Sci. 67 (1), 28-40. http://dx.doi.org/10.1093/gerona/glr010.

Moher, D., Liberati, A., Tetzlaff, J., Altmann, D.G., 2009. Preferred reporting items for systematic reviews and meta-analyses: the PRISMA statement. Ann. Intern. Med. 151 (4), 264-269.

Molina, R., Denadai, B.S., 2012. Dissociated time course recovery between rate of force development and peak torque after eccentric exercise. Clin. Physiol. Funct. Imaging 32 (3), 179-184. http://dx.doi.org/10.1111/j.1475-097X.2011.01074.x.

Moritani, T., DeVries, H.A., 1979. Neural factors versus hypertrophy in the time course of muscle strength gain. Am. J. Phys. Med. 58 (3), 115-130.

de Oliveira, F.B., Rizatto, G.F., Denadai, B.S., 2013. Are early and late rate of force development differently influenced by fast-velocity resistance training? Clin. Physiol. Funct. Imaging 33 (4), 282-287. http://dx.doi.org/10.1111/cpf.12025.

Oliveira, F.B., Oliveira, A.S., Rizatto, G.F., Denadai, B.S., 2013. Resistance training for explosive and maximal strength: effects on early and late rate of force development. J. Sports Sci. Med. 12 (3), 402-408.

Peterson, M.D., Rhea, M.R., Sen, A., Gordon, P.M., 2010. Resistance exercise for muscular strength in older adults: a meta-analysis. Ageing Res. Rev. 9 (3), 226-237. http://dx. doi.org/10.1016/j.arr.2010.03.004.

Reeves, N.D., Narici, M.V., Maganaris, C.N., 2004. In vivo human muscle structure and function: adaptations to resistance training in old age. Exp. Physiol. 89 (6), 675-689.

Rothstein, H., Sutton, A., Borenstein, M., 2005. Publication Bias in meta-analysis. The Trim and Fill Method. Wiley, West Sussex.

Rubenstein, L.Z., 2006. Falls in older people: epidemiology, risk factors and strategies for prevention. Age Ageing 35 (S2), ii37-ii41. http://dx.doi.org/10.1093/ageing/afl084.

Sayers, S.P., Gibson, K., Bryan, J.M., 2016. Improvement in functional performance with high-speed power training in older adults is optimized in those with the highest training velocity. Eur. J. Appl. Physiol. 116 (11-12), 2327-2336. http://dx.doi.org/ 10.1007/s00421-016-3484-x.

Scaglioni, G., Ferri, A., Minetti, A.E., Martin, A., Van Hoecke, J., Capodaglio, P., Sartorio, A., Narici, M.V., 2002. Plantar flexor activation capacity and $\mathrm{H}$ reflex in older adults: adaptations to strength training. J. Appl. Physiol. 92 (6), 2292-2302.

Silva, N.L., Oliveira, R.B., Fleck, S.J., Leon, A.C., Farinatti, P., 2014. Influence of strength training variables on strength gains in adults over 55 years-old: a meta-analysis of dose-response relationships. J. Sci. Med. Sport 17 (3), 337-344. http://dx.doi.org/10. 1016/j.jsams.2013.05.009.

Simoneau, E., Martin, A., Porter, M.M., Van Hoecke, J., 2006. Strength training in old age: adaptation of antagonist muscles at the ankle joint. Muscle Nerve 33 (4), 546-555.

Stewart, V.H., Saunders, D.H., Greig, C.A., 2014. Responsiveness of muscle size and 
strength to physical training in very elderly people: a systematic review. Scand. J. Med. Sci. Sports 24 (1), e1-10. http://dx.doi.org/10.1111/sms.12123.

Straight, C.R., Lindheimer, J.B., Brady, A.O., Dishman, R.K., Evans, E.M., 2016. Effects of resistance training on lower-extremity muscle power in middle-aged and older adults: a systematic review and meta-analysis of randomized controlled trials. Sports Med. 46 (3), 353-364. http://dx.doi.org/10.1007/s40279-015-0418-4.

Sundstrup, E., Jakobsen, M.D., Andersen, L.L., Andersen, T.R., Randers, M.B., Helge, J.W., Suetta, C., Schmidt, J.F., Bangsbo, J., Krustrup, P., Aagaard, P., 2016. Positive effects of 1-year football and strength training on mechanical muscle function and functional capacity in elderly men. Eur. J. Appl. Physiol. 116 (6), 1127-1138. http://dx.doi.org/ 10.1007/s00421-016-3368-0.

Van Cutsem, M., Duchateau, J., Hainaut, K., 1998. Changes in single motor unit behaviour contribute to the increase in contraction speed after dynamic training in humans. J. Physiol. 513 (Pt 1), 295-305.

Vandenbogaerde, T.J., Hopkins, W.G., 2011. Effects of acute carbohydrate supplementation on endurance performance: a meta-analysis. Sports Med. 41 (9), 773-792. http://dx.doi.org/10.2165/11590520-000000000-00000.

Young, W.B., Bilby, G.E., 1993. The effect of voluntary effort to influence speed of contraction on strength, muscular power, and hypertrophy development. J. Strength Cond. Res. 7 (3), 172-178. 